\title{
Crafting Positive/Negative Patterns and Nanopillars of Polymer Brushes by Photocatalytic Lithography
}

Guido Panzarasa, Guido Soliveri, Silvia Ardizzone

\section{HIGHLIGHTS}

Photocatalytic lithography is demonstrated as an efficient and versatile patterning strategy.

Both positive and negative patterns of polymer brushes are obtained using direct photocatalytic lithography.

Nanosphere lithography coupled to remote photocatalysis allows to achieve polymer pillars patterns with nanoscale size.

\section{KEYWORDS}

Polymer brushes, Titanium dioxide, Photocatalytic lithography, Patterning, Nanosphere lithography, Nanopillars, SI-ATRP 


\section{ABSTRACT}

We demonstrate how micro- and nanostructures of polymer brushes can be prepared using photocatalytic lithography as a convenient and versatile approach. Both positive and negative micro-patterns of polymer brushes are obtained by patterning self-assembled monolayers through direct photocatalytic lithography. Such approach mimics conventional photolithography but is free from the disadvantages associated to the use of polymeric resists. Moreover, for the first time, the ability to generate nanometer-sized pillars of polymer brushes using remote photocatalysis coupled with nanosphere lithography is demonstrated. Our results confirm the great potentialities of photocatalytic lithography for efficient patterning of polymer brushes.

\section{Introduction}

Patterning is one of the keywords of contemporary surface engineering. This is especially true for the field of polymer brushes, ensembles of surface-tethered polymer chains, which, due to their high grafting density, maintain a considerably stretched conformation. ${ }^{1,2}$ Their fast response to environmental changes makes polymer brushes the first choice to develop stimuli-responsive coatings. Making patterned polymer brushes with different functionalities (e.g. antifouling features or $\mathrm{pH}-$, ion-, light- and thermo-responsiveness) a dramatic modification of the surface properties can be achieved. Successful examples of such approach include, but are not limited to, self-cleaning surfaces, microfluidic devices, sensors and actuators. ${ }^{3-8}$

Progresses in surface-initiated polymerization (SIP) made the production of patterned polymer brushes a straightforward process in which a pre-patterned initiator monolayer, grafted on the surface, is amplified from a two-dimensional (2D) pattern into a three-dimensional (3D) brush structure by SIP. ${ }^{9}$ Such approach is called "grafting-from", as opposed to "grafting-to" which relies on the attachment of pre-made polymer chains, and is nowadays the election method to obtain 
polymer brushes. Surface-initiated atom transfer radical polymerization (SI-ATRP) is one of the most used SIP techniques, allowing the growth of well-defined brushes under mild conditions. In this technique, a lower-oxidation-state metal (typically copper) complex abstracts a halogen atom from an alkyl halide (typically a secondary or tertiary one) to generate a radical center and in turn is oxidized. The subsequent addition of monomers then starts the chain growth. In activators regenerated by electron transfer (ARGET) ATRP excess reducing agents such as ascorbic acid are used to continuously reduce the oxidized metal complex to its active form: in this way the concentration of metal complex required can be reduced down to ppm amounts. ${ }^{10}$ Patterning of surface-grafted initiator molecules can be accomplished using a number of techniques, which can be split in two major groups ${ }^{11}$ : additive (e.g. microcontact printing) or subtractive (lithographic techniques). The latter presents many variations ${ }^{12}$, ranging from conventional photolithography to electron/ion beam lithography, scanning probe lithography, nanosphere lithography and photocatalytic lithography. The photocatalytic approach is the core of the present work.

When a photoactive oxide, such as titanium dioxide, is irradiated by photons with higher energy compared the oxide band gap, electron-hole pairs are produced. Electrons are good reducing agents and holes powerful oxidizing agents; both can react with adsorbed molecules (e.g. water and oxygen molecules) leading to formation of highly reactive intermediates, which in turn degrade organic molecules. ${ }^{13-15}$ This is the suggested working principle of photocatalytic lithography, which can be performed both in a "direct" and a "remote" fashion. ${ }^{16-18}$ In direct photocatalytic lithography, as the name suggests, the pattern is obtained directly on the surface of titanium dioxide by irradiation with UV light through a photomask. If the titania surface is covered with an organic monolayer, selective degradation of the latter will be obtained in the UV-exposed zones. Remote photocatalytic lithography, on the other hand, it is based on the migration of photogenerated reactive oxygen species from the titania surface to the surrounding atmosphere, even for distances $>100 \mu \mathrm{m}$. As suggested by Kubo et al. ${ }^{19}, \mathrm{H}_{2} \mathrm{O}_{2}$ molecules, which are generated at the photoactive surface from atmospheric water and oxygen, migrate in the surrounding air and are cleaved to $\mathrm{HO}^{\bullet}$ 
radicals in the radiation exposed areas, e.g. on the substrate to be patterned. Compared to traditional photolithography, photocatalytic lithography does not need specific photoresists, nor does it require dedicated apparatus as in the case of beam and scanning probe lithography. Moreover, compared to conventional UV-lithography, it does not require powerful (and potentially dangerous) light sources. $^{18}$

We reported, in a recent paper $^{20}$, the first successful application of both direct and remote photocatalytic lithography as an innovative, easy and versatile technique for micro-patterning of polymer brushes. The obtained results prompted us to undergo a systematic exploration of the opportunities offered by such technique for the micropatterning of functional, stimuli-responsive polymer brushes. ${ }^{21}$ Noteworthy, the absence of surface contaminants such as resist residues (typical of conventional lithography) allowed us to perform accurate electrochemical measurements on the obtained patterns. ${ }^{22}$

The cheapness and user-friendliness of this lithographic approach, along with the promising applications of microstructured polymer brushes, force us to investigate what kind of patterns could be obtained and especially to push the limit of our technique towards miniaturization. Here, we show how, by direct photocatalytic lithography, both positive and negative photomask replicas can be obtained as in conventional photolithography. Moreover, we demonstrate that patterns of polymer nanopillars with different sizes appear when remote photocatalytic lithography is coupled with nanosphere lithography. That is even the first report of nanometer-sized structures obtained by remote photocatalysis.

\section{Materials and methods}

All chemicals and solvents were purchased from Sigma-Aldrich and used as received, unless otherwise stated. Inhibitor-free methyl methacrylate (MMA) was prepared by passing it through an 
inhibitor-remover column and storing at $+4{ }^{\circ} \mathrm{C}$. Silres BS 1701 (Wacker Chemie AG) is a mixture of isomeric octyltriethoxysilanes with iso-octyltriethoxysilane as the main component. The ATRP initiator-bearing silane (3-(2-bromoisobutyramido)propyl)triethoxysilane (BIB-APTES) was prepared and used to functionalize titania and silicon surfaces according to our procedure. ${ }^{20-23}$. Titanium dioxide-coated silicon substrates and glass slides were obtained by electrochemicallyassisted deposition as previously reported ${ }^{24}$. TEM grids (Gilder Grids, nickel, d $3.05 \mathrm{~mm}$, square mesh) were used as photomasks ${ }^{20}$. A Jelosil HG500 halogen lamp (230 V, $500 \mathrm{~W}$, effective power density between 280 and $400 \mathrm{~nm}$ from a distance of $40 \mathrm{~cm}: 57.5 \mathrm{~mW} \mathrm{~cm}^{-2}$ ) was used as a UV source.

\subsection{Synthesis of silica nanoparticles}

Silica nanospheres were synthesized using a previously reported Stöber method ${ }^{25,26}$ by the hydrolysis and condensation of tetraethoxysilane (TEOS) with basic catalysis. The following concentrations of reactants were employed: $[$ TEOS $]=0.27 \mathrm{M},\left[\mathrm{NH}_{3}\right]=2 \mathrm{M}$ and $\left[\mathrm{H}_{2} \mathrm{O}\right]=7 \mathrm{M}$. The appropriate amounts of water and ammonium hydroxide (concentrated aqueous solution, $16 \mathrm{M}$ according to titration) were diluted with ethanol to a volume of $320 \mathrm{~mL}$ in a in a $500 \mathrm{~mL}$ roundbottomed flask equipped with an Allihn condenser and a mechanical stirrer. The solution was heated to $30{ }^{\circ} \mathrm{C}$ in an oil bath under stirring $(200 \mathrm{rpm})$ and then $20 \mathrm{~mL}$ of TEOS were added at once. After $2.5 \mathrm{~h}$, the resulting suspension was concentrated by rotary evaporation and the particles were collected by three cycles of centrifugation $(5000 \mathrm{rpm}, 15 \mathrm{~min}$ ) and redispersion in ethanol. The final concentration of the suspension, determined gravimetrically, was $134 \mathrm{mg} \mathrm{mL}^{-1}$.

\subsection{Particle characterization}

Dynamic light scattering (DLS) and Scanning electron microscopy (SEM) were employed to analyze the size distribution of the obtained silica nanoparticles. DLS measurements were performed using a Malvern Zetasizer Nano ZS with a fixed scattering angle of $173^{\circ}$, a He-Ne laser 
and DLS software for Windows (version 6.11, Malvern). The analyses were performed at $25^{\circ} \mathrm{C}$ on

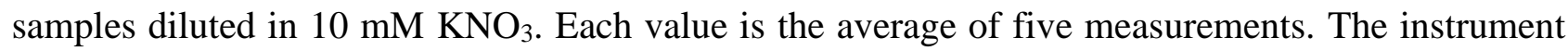
was checked with standard polystyrene latex with a diameter of $100 \mathrm{~nm}$. SEM measurements were performed using a Jeol JSM 7600f Schottky Field Emission Scanning Electron Microscope. To prepare the samples for SEM imaging, a drop of suspension was spread and allowed to dry onto a silicon substrate previously cleaned by piranha treatment (i.e. hydrophilic). From 200 to 250 individual particle diameters were measured for each sample using the ImageJ processing program.

\subsection{Polymer brushes by surface-initiated ARGET ATRP}

An optimized activators regenerated by electron transfer (ARGET) ATRP protocol was selected for the growth of polymer brushes. ${ }^{20}$ In a nitrogen-purged Schlenk flask, $0.021 \mathrm{~g}(\sim 0.07 \mathrm{mmol})$ of tris(2-pyridylmethyl)amine (TPMA), $0.002 \mathrm{~g}$ ( 0.01 mmol) of copper(II) bromide and $0.023 \mathrm{~g}(0.13$ mmol) of ascorbic acid were dissolved in $10 \mathrm{~mL}$ of a $4: 1 \mathrm{v} / \mathrm{v}$ methanol-water mixture previously degassed by nitrogen bubbling. $10 \mathrm{~mL}(94 \mathrm{mmol})$ of degassed MMA were added and the mixture was stirred under nitrogen. A $5 \mathrm{~mL}$-aliquot of this mixture was poured under nitrogen over the initiator-functionalized substrates placed each in a Schlenk flask. Polymerization proceeded at 30 ${ }^{\circ} \mathrm{C}$ for $4 \mathrm{~h}$; then the samples were rinsed extensively with THF, gently sonicated in the same solvent and dried under a nitrogen stream.

2.4 Generation of positive and negative patterns of polymer brushes by direct photocatalytic lithography

To generate a positive pattern of polymer brushes, BIB-APTES-functionalized titania substrates were exposed to UV light for $1 \mathrm{~h}$ using a TEM grid as a contact photomask. The resulting positive pattern was amplified into polymer brushes by the ARGET ATRP of MMA for $4 \mathrm{~h}$. The mean brush thickness, measured on a non-patterned sample was $120 \pm 2 \mathrm{~nm}$. 
To obtain the corresponding negative pattern, titania substrates were first functionalized with a selfassembled monolayer of alkylsiloxanes via the chemical vapor deposition (CVD) of Silres BS 1701 at $100{ }^{\circ} \mathrm{C}$ according to the procedure of Soliveri et al. ${ }^{27}$ To generate the pattern, the alkylsiloxanefunctionalized substrate was irradiated with UV light for $1 \mathrm{~h}$ through a TEM grid as a contact photomask. The substrate was functionalized with the BIB-APTES initiator and the pattern amplified into polymer brushes as described before (see Scheme 1).

\subsection{Generation of polymer brushes nanopillars by coupling remote photocatalysis with nanosphere} lithography

Particle self-assembled monolayers were obtained by means of spin-coating. The silica particle suspension was diluted with ethanol to a concentration of $1 \% \mathrm{~m} / \mathrm{v}$. A $0.5 \% \mathrm{v} / \mathrm{v}$ of $\alpha$-terpineol was added to improve self-assembly. About $20 \mu \mathrm{L}$ of the diluted particle suspension were spread on the surface of a BIB-APTES-functionalized silicon substrate and spin-coated (500 rpm, $100 \mathrm{rpm} \mathrm{s}^{-1}$, $20 \mathrm{~s})$.

The substrates were then subjected to remote photocatalytic lithography using the particles monolayer as photomasks. A titania-coated glass slide facing the substrate to be patterned, separated by a $100-\mu \mathrm{m}$ thick Teflon ${ }^{\circledR}$ spacer, was irradiated with UV light for $5 \mathrm{~h}$. The particles were then removed by gentle sonication in ethanol, the substrates dried under nitrogen and the obtained pattern amplified with PMMA brushes as described before.

\subsection{Characterization of the patterns of polymer brushes}

Optical microscopy images and high resolution FTIR spectra were acquired with a Nicolet ${ }^{\mathrm{TM}}$ $\mathrm{iN}^{\mathrm{TM}} 10$ Infrared Microscope in reflectance mode, using a liquid nitrogen-cooled MCT detector (spectral range 4000-675 $\mathrm{cm}^{-1}$, resolution $4 \mathrm{~cm}^{-1}$, aperture $150 \times 150 \mu \mathrm{m}$ ). Background (500 scans) was collected before each sample (1000 scans) from cleaned silicon or titanium dioxide. The acquisition software performed baseline correction automatically. Water contact angle was measured using a Krüss Easy Drop Standard with DSA1 software. A $3 \mu$ L-drop of HPLC-grade 
water was deposited and the contact angle measured after $5 \mathrm{~s}$. Each value is the average of at least three measurements performed on different spots of a same sample. Brush thickness was measured using a Filmetrics F20 reflectometer. Scanning electron microscopy (SEM) was performed using a Jeol JSM 7600f Schottky Field Emission Scanning Electron Microscope. Atomic force microscopy (AFM) pictures were acquired using a SPM Bruker Dimension Icon, in air at room temperature with the Peak Force Tapping technique.

\section{Results and discussion}

3. Positive and negative patterns of polymer brushes obtained using direct photocatalytic lithography

In our previous reports ${ }^{20-22}$, we described how the positive replicas of a photomask could be amplified as polymer brushes by means of direct photocatalytic lithography (Scheme 1a). Innovative findings reported here demonstrate how we were able to obtain the corresponding negative pattern using a sacrificial SAM (Scheme 1b). First, alkylsilanes, inert towards ATRP, were grafted on the surface of titanium dioxide in such a way to generate a monolayer, where it acts as a "negative-tone resist", using a term from conventional photolithography. The alkylsiloxane layer was patterned using the direct photocatalytic lithography, or rather, a mask (a Ni TEM grid) was settled down on the sample and we irradiated it for one hour under 365 nm-radiation. 
a)
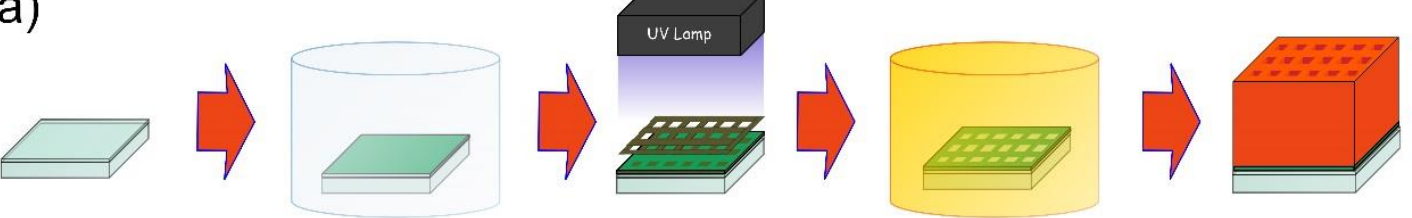

$\mathrm{TiO}_{2}$ layer on substrate

$$
\begin{aligned}
& \text { Initiator } \\
& \text { grafting }
\end{aligned}
$$

Initiator selective degradation

Polymerization

b)

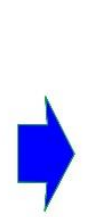

$\mathrm{TiO}_{2}$ layer on substrate

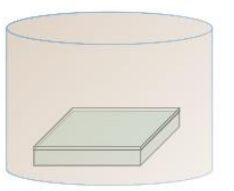

Hydrophobic silane monolayer deposition

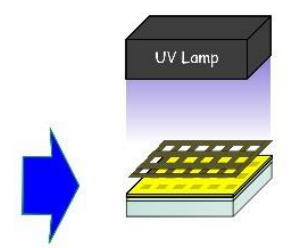

\section{Silane selective} degradation
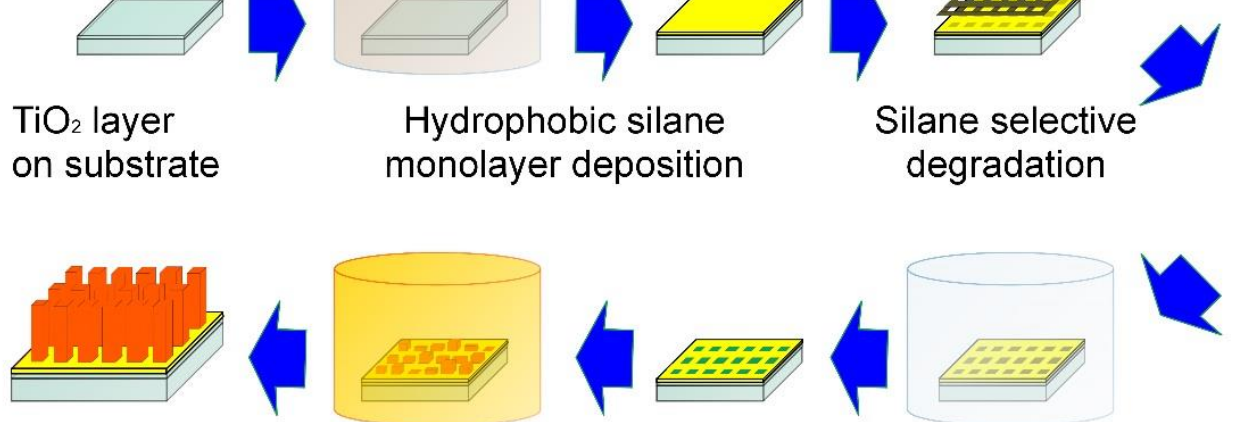

Polymerization

Initiator grafting

Supported titanium dioxide layer $\square$ Polymerization initiator layer $\square$ Hydrophobic silane layer

Polymer brushes

Scheme 1. Depiction of the processes employed to obtain positive (a) and negative (b) patterns of polymer brushes by direct photocatalytic lithography.

As shown by the FTIR spectrum (Figure S1) and the lowering of the water contact angle from $100^{\circ}$ to $0^{\circ}$, the zones which have been $\mathrm{UV}$-exposed are free from organic contamination and are thus prone to be filled with another grafting molecule, i.e. BIB-APTES, an initiator of polymerization. The corresponding negative pattern made of polymer brushes is successfully achieved by performing SI-ATRP, as judged by optical microscopy (Figure S2) and atomic force microscopy (Figure 1). 
a)

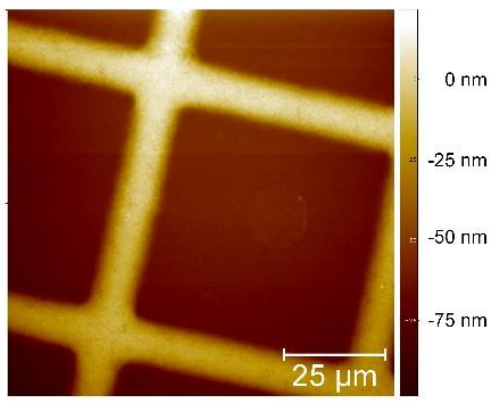

d)

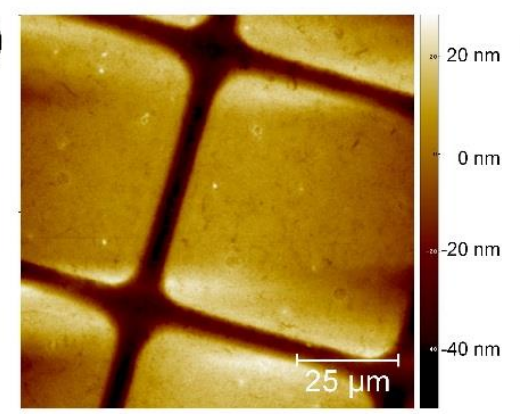

b)

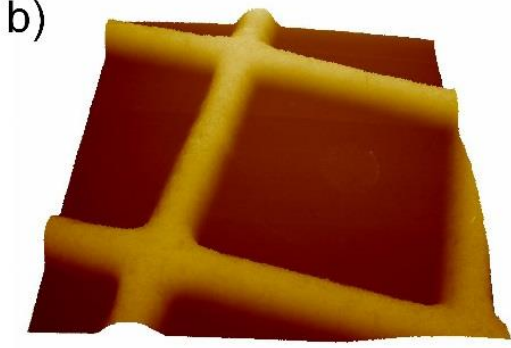

e)

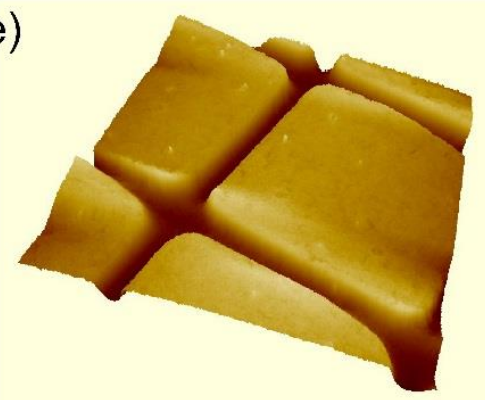

c)

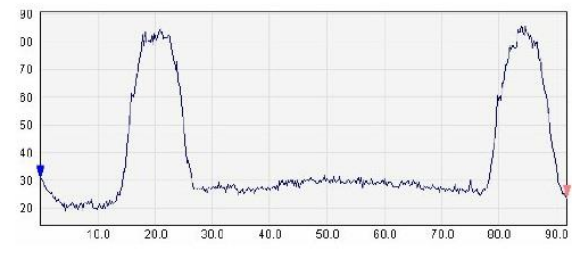

f)

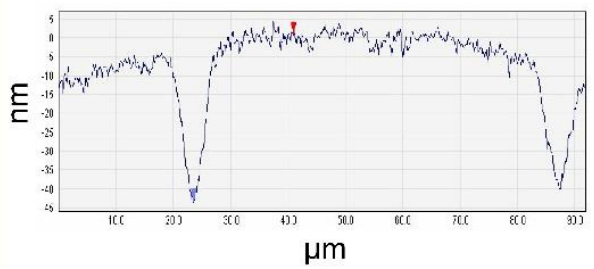

Figure 1. AFM images of the positive $(a, b, c)$ and negative $(d, e, f)$ patterns of polymer brushes obtained by direct photocatalytic lithography. Respectively topography (tapping mode) picture, 3D picture and profilometry of a section.

The quality of the alkylsiloxane layer is of paramount importance for the resolution of the polymer brushes pattern: it must be tightly packed and homogeneous to reduce the risk of spurious polymerization due to the formation of islands during the successive deposition of the BIB-APTES initiator. The chemical vapor deposition (CVD) of Silres BS 1701 proved to be a successful approach. $^{27}$

\subsection{Coupling remote photocatalysis with nanosphere lithography}

One of the main advantages of remote photocatalytic lithography is that it can be performed on virtually any kind of substrate. The patterning agents, the reactive oxygen species generated on a titania surface, diffuse towards the substrate where their action has to be exploited. This allows to experiment with different kind of photomasks, among which the most interesting is a selfassembled layer of micro- or nanospheres like those used to perform nanosphere lithography (NSL). NSL attracted great interest as a simple, bench-top and materials-general approach to obtain periodic particle nanostructures using ordered arrays of polymer or silica spheres as lithographic 
and evaporation masks. NSL allows to produce well-ordered 2D periodic arrays of nanostructures from a wide variety of materials (including polymer brushes ${ }^{28}$ ) on many substrates. ${ }^{29}$ For example, nanosphere lithography has been reported to fabricate nanohole arrays to be used as replication molds for the casting of polymer pillars. ${ }^{30}$ These structures have already been exploited for the development of smart surfaces thanks to their peculiar wetting behavior, e.g. to allow easier harvesting of cultured cells. ${ }^{31}$

Here, by using a self-assembled monolayer of particles as a photomask, we coupled remote photocatalysis with nanosphere lithography (Scheme 2) and demonstrated not only the scalable formation of polymer brushes pillars but also, more importantly, that remote photocatalytic lithography has the potential to reach nanometer-scale resolution patterns.

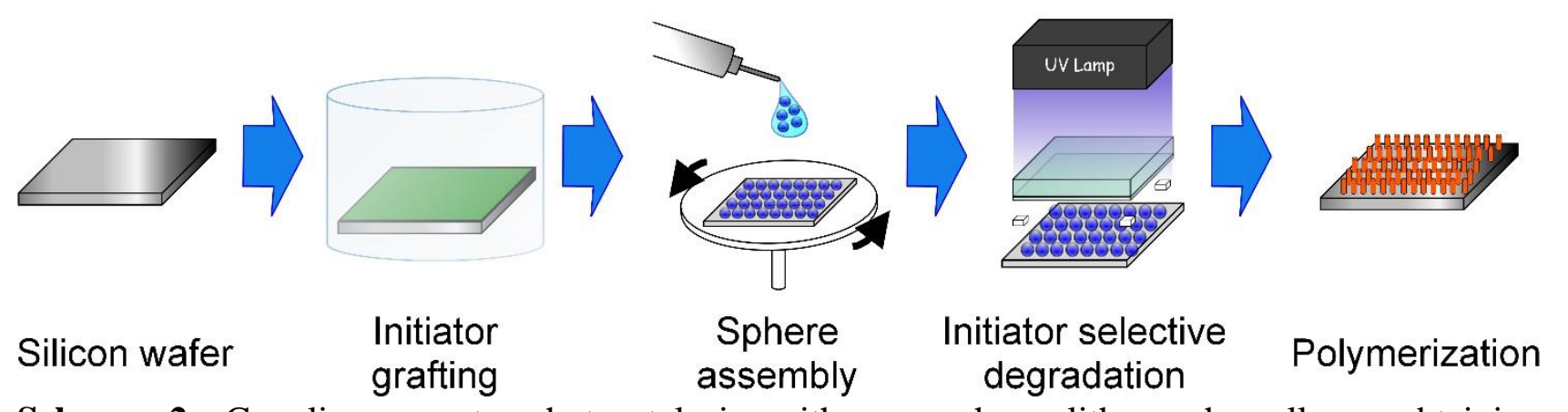

Scheme 2. Coupling remote photocatalysis with nanosphere lithography allows obtaining nanopillars of polymer brushes.

Silica particles (PCS diameter $609 \pm 25 \mathrm{~nm}$, SEM diameter $562 \pm 16 \mathrm{~nm}$ ) with very good monodispersity (PdI 0.071, according to PCS) were synthesized and used to assemble the photomask (Figure 2b).

From the morphological analysis, it can be deduced that, after SI-ATRP, arrays of polymer brushes shaped as conical pillars emerging from a polymer "carpet" are obtained (Figure 2d,e). According to SEM images, the mean diameter of the polymer pillars is $250 \pm 25 \mathrm{~nm}$ while the mean linear distance between two adjacent polymer pillars is $268 \pm 29 \mathrm{~nm}$, (Figure 2c).

We hypothesize that this peculiar morphology would originate from an anisotropic, gradient-like etching due to the diffusion of photocatalytically-generated ROS under the particles, and 
consequently due to lateral oxidation phenomena. ${ }^{18}$ According to the mechanism depicted in Figure 2a, the pillars would arise from the zones where the particles actually touch the substrate, efficiently screening the underlying initiator molecules from the etching action of ROS, while the "carpet" would originate from inter-particle spaces.
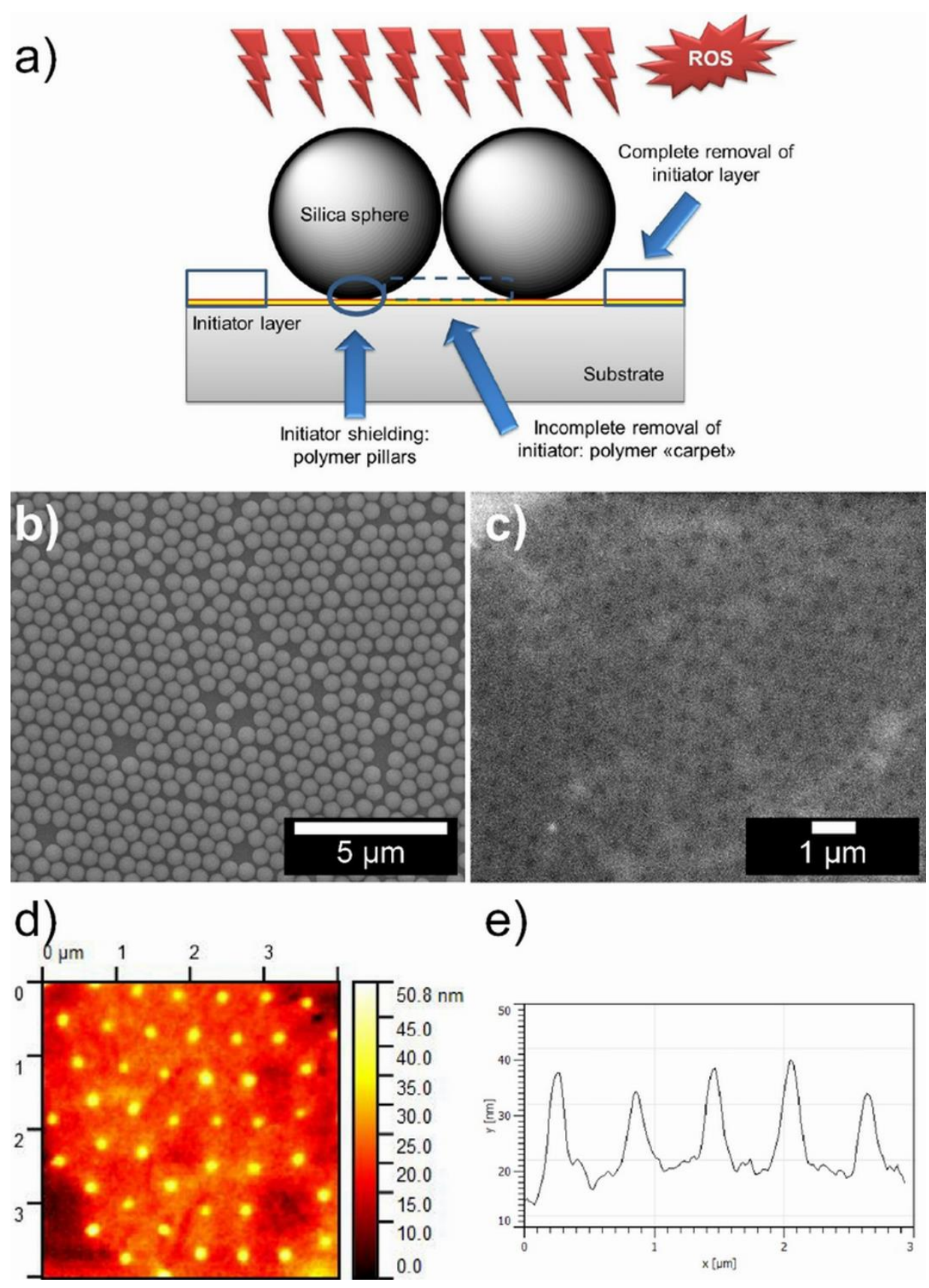

e)

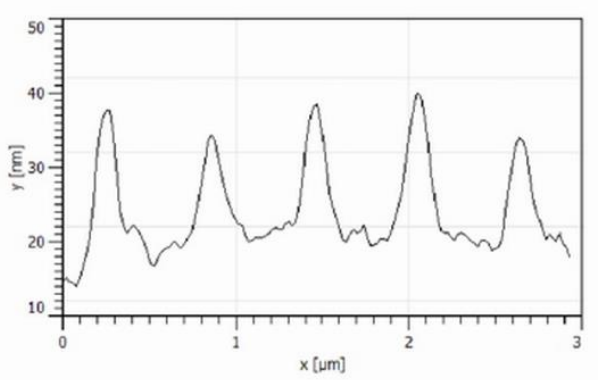

Figure 2. Remote photocatalysis coupled with nanosphere lithography. a) Proposed mechanism for the generation of the structures obtained after pattern amplification with polymer brushes. b) SEM image of the silica nanosphere photomask assembled on the initiator-coated substrate. SEM (c) and AFM $(d, e)$ images of the pattern obtained after $5 \mathrm{~h}$ of remote photocatalysis followed by surfaceinitiated polymerization. 


\section{Conclusions}

Polymer brushes represent a crucial tool in modern surface chemistry and material science. In this context, the possibility to use selectively activated surfaces to fabricate controlledand scalable patterned polymer brushes has been widely reported to have a strong impact in the next future. Notwithstanding the high relevance and the proved effectiveness of photocatalytic lithography, just few reports exploit the knowledge of inorganic photocatalysis in the field of polymer brushes. We strongly think that hotocatalysis has much to give to polymer brushes. For this reason, we employed the competences successfully collected in the last years to test the malleability and the limits of this promising approach. In this paper, first we showed how, by adding few simple steps to the classical direct photocatalytic lithography, negative patterns can be easily obtained. Moreover, the combination of nanosphere lithography, remote photocatalysis and polymer brush synthesis enabled the production of highly ordered and tunable nano-sized structures (pillars). The dimension and position of these polymer brush nano-pillars can be adjusted by changing the dimension and shape of the self-assembled particle mask.

We are confident that our results are not only a solid proof of the versatility of photocatalytic lithography as a patterning strategy but can open new concrete possibilities for the development of next-generation nanostructured hybrid surfaces, with two or more different functionalities arranged in a predetermined texture.

\section{Acknowledgements}

It is the authors' desire to express their greatest gratitude for Dr. Gianluigi Marra and Dr. Alberto Savoini (Eni Donegani Research Center for Renewable Energies and Environment, Novara, Italy) for performing the SEM and AFM analyses, respectively. G. S. gratefully acknowledges Dr. Stephanie Hoeppener for her precious teachings . 


\section{Appendix A. Supplementary data}

\section{References}

(1) Chen, T.; Amin, I.; Jordan, R. Chem. Soc. Rev. 2012, 41, 3280-3296.

(2) Barbey, R.; Lavanant, L.; Paripovic, D.; Schüwer, N.; Sugnaux, C.; Tugulu, S.; Klok, H.-A. Chem. Rev. 2009, 109, 5437-5527.

(3) Luzinov, I.; Minko, S.; Tsukruk, V. V. Soft Matter, 2008, 4, 714.

(4) Minko, S. Polym. Rev. 2006, 46, 397-420.

(5) Ayres, N. Polymer Chemistry, 2010, 1, 769-777.

(6) Azzaroni, O. J. Polym. Sci. Part A Polym. Chem. 2012, 50, 3225-3258.

(7) Krishnamoorthy, M.; Hakobyan, S.; Ramstedt, M.; Gautrot, J. E. Chem. Rev. 2014, 114, 10976-11026.

(8) Becer, C. R.; Haensch, C.; Hoeppener, S.; Schubert, U. S. Small 2007, 3, 220-225.

(9) Jia, H.; Wildes, A.; Titmuss, S. Macromolecules 2012, 45, 305-312.

(10) Matyjaszewski, K.; Dong, H.; Jakubowski, W. Langmuir 2007, 23, 4528-4531.

(11) Biswas, A.; Bayer, I. S.; Biris, A. S.; Wang, T.; Dervishi, E.; Faupel, F. Adv. Colloid Interface Sci. 2012, 170, 2-27.

(12) Ito, T.; Okazaki, S. Nature 2000, 406, 1027-1031.

(13) Carp, O. Prog. Solid State Chem. 2004, 32, 33-177.

(14) Diebold, U. Surf. Sci. Rep. 2003, 48, 53-229.

(15) Nakata, K.; Fujishima, A. J. Photochem. Photobiol. C Photochem. Rev. 2012, 13, 169-189.

(16) Kubo, W.; Tatsuma, T.; Fujishima, A.; Kobayashi, H. J. Phys. Chem. B 2004, 108, 30053009.

(17) Tatsuma, T.; Kubo, W.; Fujishima, A. Langmuir 2002, 18, 9632-9634.

(18) Paz, Y. Beilstein J. Nanotechnol. 2011, 2, 845-861. 
(19) Kubo, W.; Tatsuma, T. J. Am. Chem. Soc. 2006, 128, 16034-16035.

(20) Panzarasa, G.; Soliveri, G.; Sparnacci, K.; Ardizzone, S.; Chem. Commun. 2015, 51, 73137316.

(21) Panzarasa, G.; Soliveri, G.; Ardizzone, S.; Sparnacci, K. Mater. Today Proc. 2015, 2, 41834189.

(22) Panzarasa, G.; Soliveri, G.; Pifferi, V. J. Mater. Chem. C 2015.

(23) Panzarasa, G.; Aghion, S.; Soliveri, G. Nanotechnology 2016, 27, 02 LT03.

(24) Maino, G.; Meroni, D.; Pifferi, V.; Falciola, L.; Soliveri, G.; Cappelletti, G.; Ardizzone, S. J. Nanoparticle Res. 2013, 15, 2087.

(25) Grojo, D.; Boarino, L.; De Leo, N.; Rocci, R.; Panzarasa, G.; Delaporte, P.; Laus, M.; Sparnacci, K. Nanotechnology 2012, 23, 485305.

(26) Ravera, M.; Perin, E.; Gabano, E.; Zanellato, I.; Panzarasa, G.; Sparnacci, K.; Laus, M.; Osella, D. J. Inorg. Biochem. 2015, 151, 132-142.

(27) Soliveri, G.; Pifferi, V.; Annunziata, R.; Rimoldi, L.; Aina, V.; Cerrato, G.; Falciola, L.; Cappelletti, G.; Meroni, D. J. Phys. Chem. C 2015, 119, 15390-15400.

(28) Chen, T.; Chang, D. P.; Jordan, R.; Zauscher, S. Beilstein J. Nanotechnol. 2012, 3, 397-403.

(29) Hulteen, J. C. J. Vac. Sci. Technol. A Vacuum, Surfaces, Film. 1995, 13, 1553.

(30) Kuo, C.; Chueh, D.; Chen, P. J. Nanobiotechnology 2014, 12, 54.

(31) Hu, W.; Crouch, A. S.; Miller, D.; Aryal, M.; Luebke, K. J. Nanotechnology 2010, 21, 385301 . 